International Journal of Pure and Applied Mathematics

Volume 83 No. 2 2013, 261-270

ISSN: 1311-8080 (printed version); ISSN: 1314-3395 (on-line version)

url: http://www.ijpam.eu

doi: http://dx.doi.org/10.12732/ijpam.v83i2.5

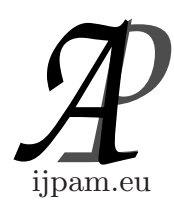

\title{
A CANTOR $p$-ARY DECOMPOSITION ON THE HILBERT CUBE
}

\author{
Aniruth Phon-On \\ Prince of Songkla University \\ Pattani Campus \\ Pattani, 94000, THAILAND \\ and \\ Centre of Excellence in Mathematics
}

CHE, Si Ayutthaya Rd., Bangkok, 10400, THAILAND

Abstract: Given a positive odd integer $p$ with $p \geq 3$, the Cantor $p$-ary $C_{p}$ set and the Cantor $p$-ary function $f_{p}$ are constructed. $C_{p}$ is a generalization of the Cantor set in the case that the measure of the set $C_{p}$ is still zero and $f_{p}^{\infty}$ defined on the Hilbert Cube $Q$ is a generalization of the Cantor function. Also, for any $s \in(0,1)$, let

$$
G_{f_{p}^{\infty}}^{s}=\left\{\{s\} \times\left(f_{p}^{\infty}\right)^{-1}(c) \mid c \in Q_{2}\right\}
$$

and $S$ is the set of all singletons in $([0, s) \cup(s, 1]) \times Q_{2}$. Then $G=G_{f_{p}^{\infty}}^{s} \cup S$ is an upper semi continuous decomposition on the Hilbert Cube $Q$. Moreover, $Q / G$ is homeomorphic to $Q$.

AMS Subject Classification: 54C50

Key Words: decomposition, upper semi continuous, Cantor $p$-ary set

\section{Introduction}

A search through literatures about the cantor set and cantor function yields a well-known construction of the standard cantor set $C$ and the standard Cantor

Received: September 1, 2012

(C) 2013 Academic Publications, Ltd. url: www.acadpubl.eu 
function $f$, see [2], [5], [6]. The purpose of this pater is to construct the Cantor $p$-ary set and the Cantor $p$-ary function where $p$ is an any odd integer with $p \geq 3$. Although the ideas of their constructions in the paper are predictable, it can rarely be found in any papers. It is thus obvious that the Cantor $p$-ary set $C_{p}$ and the Cantor $p$-ary function $f_{p}$ are the generalization of the standard cantor set $C$ and the standard Cantor function $f$, respectively.

In [7], Garity used the map $f^{k}$, where $f^{k}$ is the product of the standard Cantor function $f$, to construct the decomposition $G$ of $\mathbb{R}^{n}$ and then showed that $\mathbb{R}^{n} / G$ is homeomorphic to $\mathbb{R}^{n}$. An arising question is that "Is this still true for an infinite dimensional Hilbert Cube $Q$ ?" This paper will provide an answer to that question. That is, we will show that for any positive odd integer with $p \geq 3$, the decomposition $G$ is induced by the $f_{p}^{\infty}$ where $f_{p}^{\infty}$ is the infinite product of the Cantor $p$-ary function $f_{p}$ and show that $Q / G$ is homeomorphic to $Q$. For $p=3$, see [10].

\section{Notation and Terminology}

For the readers who are not familiar with topology, all basic topological terminology, notation, and definitions can be found in [8], [9].

Let $I$ be the interval $[0,1]$. For each $n \geq 1$, we write

$$
I^{n}=\prod_{i=1}^{n} I_{i}, \quad Q_{n+1}=\prod_{i=n+1}^{\infty} I_{i}
$$

where $I_{i}=I$. The Hilbert Cube is a countable product of $I_{i}$ where $I_{i}=[0,1]$ for all $i \geq 1$, and is denoted by $Q$. That is,

$$
Q=\prod_{i=1}^{\infty} I_{i}
$$

Also, for each $n$ we can write the Hilbert Cube as

$$
Q=I^{n} \times Q_{n+1} .
$$

A decomposition $G$ of a space $Q$ is a partition of $Q$. Associated with any decomposition $G$ of a space $Q$ is the decomposition space denoted as $Q / G$. Its topology is described by means of the decomposition map $\pi: Q \rightarrow Q / G$ sending each $q \in Q$ to the unique element of $G$ containing $q$. The topology on $Q / G$ is the quotient space topology induced by $\pi$. 
Definition 1. (see [4]) A decomposition $G$ of $Q$ is said to be upper semicontinuous(usc) if every $g \in G$ is compact and the quotient map

$$
\pi: Q \rightarrow Q / G
$$

is a closed map.

Definition 2. (see [4]) A decomposition $G$ of a space $Q$ is realized by a pseudo-isotopy if there exists a pseudo-isotopy $\Psi_{t}$ of $Q$ to $Q$ such that $\Psi_{0}=I d_{Q}$ and $G=\left\{\Psi_{1}^{-1}(x) \mid x \in Q\right\}$. By a pseudo-isotopy $\Psi_{t}$ of $Q$ to $Q$ we mean a homotopy $\Psi_{t}: Q \rightarrow Q$ such that $\Psi_{t}$ is a homeomorphism for each $t \in[0,1)$ and $\Psi_{1}$ is a closed surjection. Similarly, by an isotopy $\Psi_{t}$ of $Q$ to $Q$ we mean a homotopy $\Psi_{t}: Q \rightarrow Q$ such that $\Psi_{t}$ is a homeomorphism for each $t \in[0,1]$.

Definition 3. A closed set $\mathrm{C}$ in $\mathbb{R}^{n}$ or in an $n$-dimensional manifold is said to be cellular if there is a nested sequence $C_{1}, C_{2}, \ldots$ of $n$ cells with $C_{i+1}$ a subset of the interior of $C_{i}$ and $C=\bigcap_{i}^{\infty} C_{i}$.

Next we will define a cellularity in $Q$ which is quite similar to the definition in finite dimensional case. Here we replace the term $n$-cells by normal cubes. The definition of a normal cube and related definitions can be found in [1], [3].

Definition 4. (see [1]) Let $X$ be a closed subset of $Q . X$ is said to be a cellular subset of $Q$ if $X=\cap_{i=1}^{\infty} K_{i}$ where $K_{i+1} \subset \operatorname{int}\left(K_{i}\right)$ and $K_{i}$ is a normal cube for all $i$.

\section{Construction of a Cantor $p$-ary Set and a Cantor $p$-ary Function}

\subsection{Construction of a Cantor $p$-ary Set}

Basically, the standard Cantor set $C$ is constructed by removing middle third interval in each step, see [6], [5], [2]. We will use this idea to construct the $p$-ary Cantor set $C_{p}$ so that $C_{p} \cong C$.

Definition 5. Let $n \in \mathbb{Z}^{+}$and $p \in \mathbb{N}$ where $p$ is a positive odd integer $p$ with $p \geq 3$. Define an interval $\Theta\left(k_{1}, \ldots, k_{n}\right)$ by

$$
\Theta\left(k_{1}, \ldots, k_{n}\right)=\left[\sum_{i=1}^{i=n} \frac{k_{i}}{p^{i}}, \sum_{i=1}^{i=n} \frac{k_{i}}{p^{i}}+\frac{1}{p^{n}}\right] \text { for } k_{i} \in K_{p}
$$

where $K_{p}=\{0,1,2, \ldots, p-1\}$. 
It is clear by the definition that the length of $\Theta\left(k_{1}, \ldots, k_{n}\right)$ is $\frac{1}{p^{n}}$. Also, for each $n$,

$$
\Theta\left(k_{1}, \ldots, k_{n}\right)=\cap_{i=1}^{n} \Theta\left(k_{1}, \ldots, k_{i}\right)
$$

Let us denote the sets $K_{p}^{e}=\{0,2,4, \ldots, p-1\}$ and $K_{p}^{o}=\{1,3,5, \ldots, p-2\}$

$$
C_{n}=\left\{\Theta\left(k_{1}, k_{2}, \ldots, k_{n}\right) \mid k_{i} \in K_{p}^{e}\right\} \text {. }
$$

Let $C_{1}^{c}=\{\Theta(1), \Theta(3), \Theta(5), \ldots, \Theta(p-2)\}$, and for $n \geq 2$, define

$$
C_{n}^{c}=\left\{\Theta\left(k_{1}, \ldots, k_{n-1}, k_{n}\right) \mid k_{1}, k_{2}, \ldots, k_{n-1} \in K_{p}^{e}, k_{n} \in K_{p}^{o}\right\} .
$$

Also, let $C^{c}=\bigcup_{n=1}^{\infty}\left(\bigcup C_{n}^{c}\right)$.

Now we will use the definition of $C_{n}$ to define the Cantor $p$-ary set. That is, the Cantor $p$-ary set $C_{p}$ is defined as:

$$
C_{p}=\bigcap_{n=0}^{\infty} \bigcup C_{n}
$$

Let $\mathrm{M}$ be the Lebesque measure. Then we have the following lemma.

Lemma 6. Let $C_{p}$ be the Cantor $p$-ary set where $p$ is a positive odd integer with $p \geq 3$. Then

$$
\mathrm{M}\left(C_{p}\right)=0 .
$$

In other words, $C_{p}$ has a zero measure.

Proof. Note that for each $n$, the sum of the length of all elements $\Theta$ in $C_{n}^{c}$ is

$$
\left(\frac{p-1}{2}\right)\left(\frac{p+1}{2}\right)^{n-1}\left(\frac{1}{p^{n}}\right) .
$$

Since $C_{n}^{c} \cap C_{n+1}^{c}=\emptyset$ for all $n$, we have

$$
\mathrm{M}\left(C^{c}\right)=\sum_{n=1}^{\infty}\left(\frac{p-1}{2}\right)\left(\frac{p+1}{2}\right)^{n-1}\left(\frac{1}{p^{n}}\right)=\left(\frac{p-1}{2 p}\right)\left(\frac{1}{1-\left(\frac{p+1}{2 p}\right)}\right)=1 .
$$

But we know that

$$
C_{p} \subset\left([0,1] \backslash C^{c}\right) \bigcup \cup_{n=1}^{\infty} E_{n}
$$


where $E_{n}$ is the set of all endpoints of each interval in $C_{n}^{c}$. It is obvious that each $E_{n}$ is countable and hence $\mathrm{M}\left(\cup_{n=1}^{\infty} E_{n}\right)=0$. Thus,

$$
0 \leq \mathbf{M}\left(C_{p}\right) \leq\left([0,1] \backslash C^{c}\right) \bigcup \cup_{n=1}^{\infty} E_{n}=\left(1-\mathbf{M}\left(C^{c}\right)\right)+1=1-1+0=0 .
$$

That is, $\mathbf{M}\left(C_{p}\right)=0$.

Theorem 7. (see [11]) A compact set $X$ is homeomorphic to the standard Cantor set $C$ if and only if $X$ is totally disconnected and perfect.

Lemma 8. Let $C_{p}$ be the Cantor $p$-ary set where $p$ is an odd integer. Then $C_{p}$ is homeomorphic to $C$ where $C$ is the standard Cantor set. Moreover, each element in $C_{p}$ can be written as a $p$-ary representation consisting entirely of elements in $K_{p}^{e}$.

Proof. Note that each element in $C_{n}$ for all $n$ is compact. Since the intersection of the collection of compact sets with the non-empty finite intersection property is compact, is clear that $C_{p}$ is compact. Since the size of the components of $C_{n}$ is going to zero, this implies the totally disconnectedness of $C_{p}$. Moreover, each element of $C_{n}$ has more than two elements of $C_{n+1}$, this implies every point in $C_{p}$ is a limit point. Hence $C_{p}$ is perfect. Therefore, by Theorem 7, $C_{p}$ is homeomorphic to $C$. Next, we can see that for each $c \in C_{p}, c \in \bigcup C_{n}$ for all $n$. Thus for each $i$, there exists $k_{i}$ in $K_{p}^{e}$ so that $c \in \cap_{n=1}^{\infty} \Theta\left(k_{1}, \ldots, k_{i}\right)$. This implies that $c_{p}=\sum_{i=1}^{\infty} \frac{k_{i}}{p^{i}}$.

Remark 9. If $p=3$, then $C_{p}=C$ where $C$ is the standard Cantor set.

\subsection{Construction of a Cantor $p$-ary Function}

The Cantor $p$-ary map $f_{p}: I \rightarrow I$ is also defined as a constant on the closure of each component of $I \backslash C_{p}$ and on $C_{p}$ is defined by:

$$
f_{p}\left(\sum_{i=1}^{\infty} \frac{a_{i}}{p^{i}}\right)=\sum_{i=1}^{\infty} \frac{a_{i}}{2\left(\frac{p+1}{2}\right)^{i}},
$$

where $a_{i} \in K_{p}^{e}$.

Remark 10. Let $P$ be the set of all $\frac{p+1}{2}$-adic rationals in the closed unit interval where $p$ is an odd integer. That is,

$$
P=\left\{\frac{m}{\left(\frac{p+1}{2}\right)^{n}} \in[0,1] \mid m, n \in \mathbb{Z}\right\}
$$


1. If $c \in I$, the

$$
f_{p}^{-1}(c)= \begin{cases}1-\text { cell } & \text { if } c \in P \\ \text { singleton } & \text { if } c \notin P\end{cases}
$$

2. $\left.f_{p}\right|_{C_{p}}$ is two-to-one over the $\frac{p+1}{2}$-adic rationals in $P$;

3. $\left.f_{p}\right|_{C_{p}}$ is one-to-one over the complement of $P$;

4. $f_{p}$ itself is one-to-one over the complement of $P$.

By Remark 10, for $c \in C_{p}$,

$c$ is p-adic rational if and only if $f_{p}(c)$ is $\frac{p+1}{2}$-adic rational.

Thus, if $f_{p}(c)=\frac{m}{\left(\frac{p+1}{2}\right)^{n}}$ for some $m, n$, then by Remark 10(2), $c=\frac{2 k}{p^{n}}$ or $c=\frac{2 k+1}{p^{n}}$ for some $k$.

Let $f_{p}^{k}: I^{k} \rightarrow I^{k}$ be defined by

$$
f_{p}^{k}(x)=\left(f_{p}\left(x_{1}\right), f_{p}\left(x_{2}\right), \ldots, f_{p}\left(x_{k}\right)\right) \quad \text { for all } x \in I^{k}
$$

Note that $f_{p}^{k}$ is continuous since each component is continuous.

Lemma 11. Let $c \in I^{k}$. Then $\left(f_{p}^{k}\right)^{-1}(c)$ is either a point or a $l$-cell where $l$ corresponds to the number of $\frac{p+1}{2}$-adic rational coordinates that $c$ has, and hence $\left(f_{p}^{k}\right)^{-1}(c)$ is either a point or a $l-c e l l$.

Proof. Let $c=\left(x_{1}, \ldots, x_{k}\right) \in I^{k}$. If $c$ has no $\frac{p+1}{2}$-adic rational coordinates, then $x_{i} \notin P$ for all $i=1,2, \ldots, k$. Thus, $\left(f_{p}^{k}\right)^{-1}\left(x_{i}\right)$ is just a point in $I$ which implies that $\left(f_{p}^{k}\right)^{-1}(c)$ is a point in $I^{k}$. Next assume that the number of $\frac{p+1}{2}$-adic rational coordinates of $c$ is $l$. Denote each $b_{i}$ the $\frac{p+1}{2}$-adic rational coordinates of $c$ for $i=1, \ldots, l$. Then each $\left(f_{p}^{k}\right)^{-1}\left(b_{i}\right)$ is a 1 -cell in $I$ so $\left(f_{p}^{k}\right)^{-1}(c)$ is a $l$-cell in $I^{k}$.

Next, we will define the function $f_{p}^{\infty}: Q_{2} \rightarrow Q_{2}$. First for each $k$ define $g_{p}^{k}: Q_{2} \rightarrow Q_{2}$ by

$$
g_{p}^{k}\left(\left(x_{2}, \ldots, x_{k}, \ldots\right)\right)=f_{p}^{k}\left(\left(x_{2}, \ldots, x_{k}\right)\right) \times I d_{Q_{k+1}}\left(x_{k+1}, \ldots\right) .
$$

Thus, the function $f_{p}^{\infty}: Q_{2} \rightarrow Q_{2}$ is defined by

$$
\begin{aligned}
f_{p}^{\infty}(x) & =\lim _{k \rightarrow \infty} g_{p}^{k}(x) \\
& =\left(f_{p}\left(x_{2}\right), f_{p}\left(x_{3}\right), \ldots\right) .
\end{aligned}
$$

Since $Q_{2}$ is compact, it is obvious that $f_{p}^{\infty}$ is a closed map. 
Lemma 12. For each point $c \in Q_{2},\left(f_{p}^{\infty}\right)^{-1}(c)$ is either a point, a cell or a copy of $Q_{2}$ and the dimension of these sets corresponds to the number of $\frac{p+1}{2}$-adic rational coordinates that $c$ has.

Proof. If $c$ has no $\frac{p+1}{2}$-adic rational coordinates, it is clear that $\left(f_{p}^{\infty}\right)^{-1}(c)$ is just a point in $Q_{2}$. If $p$ has $l \frac{p+1}{2}$-adic rational coordinates, then $\left(f_{p}^{\infty}\right)^{-1}(c)$ is a $l$-cell in $Q^{2}$. If $c$ has infinitely many $\frac{p+1}{2}$-adic rational coordinates, then $\left(f_{p}^{\infty}\right)^{-1}(c)$ is a copy of $Q_{2}$.

\section{Construction of the Cantor $p$-ary Decomposition $G$}

Recall that $f_{p}^{\infty}$ is the map from $Q_{2} \rightarrow Q_{2}$. To construct a decomposition $G$ on $Q$, first we will use the function $f_{p}^{\infty}$ to define the decomposition $G_{f_{p}^{\infty}}^{s}$ on $Q^{2}$ where $Q^{2}=\{0\} \times Q_{2}$. Fix $s \in(0,1)$. Let

$$
G_{f_{p}^{\infty}}^{s}=\left\{\{s\} \times\left(f_{p}^{\infty}\right)^{-1}(c) \mid c \in Q_{2}\right\} .
$$

Lemma 13. The decomposition $G_{f_{p}^{\infty}}^{s}$ defined as above is upper semicontinuous.

Proof. This follows from the fact that $\pi_{G_{f_{p}^{s}}^{s}}=\{s\} \times f_{p}^{\infty}$. Moreover, by the Lemma $12, G_{f_{p}^{\infty}}^{s}$ is cellular.

Next will show that the decomposition $G_{f_{p}^{\infty}}^{s}$ is realized by a pseudo-isotopy.

Lemma 14. The decomposition $G_{f_{p}^{\infty}}^{s}$ is realized by a pseudo-isotopy.

Proof. Recall $f_{p}^{\infty}: Q_{2} \rightarrow Q_{2}$ is a generalized Cantor $p$-ary function in which each component is the Cantor $p$-ary function $f_{p}:[0,1] \rightarrow[0,1]$. To show that the decomposition $G_{f_{p}^{\infty}}^{s}$ is realized by a pseudo-isotopy, it suffices to show that there exists a pseudo-isotopy $\Psi_{t}$ of $Q_{s}^{2} \rightarrow Q_{s}^{2}$ such that $\Psi_{0}$ is the identity $I d_{Q_{s}^{2}}$ and $G_{f_{p}^{\infty}}^{s}=\left\{\Psi_{1}^{-1}(c) \mid c \in Q^{2}\right\}$. For $t \in[0,1]$, define $\Psi_{t}: Q_{s}^{2} \rightarrow Q_{s}^{2}$ by

$$
\Psi_{t}(s, x)=\left(s,(1-t) x+t f_{p}^{\infty}(x)\right) .
$$

It is clear that $\Psi_{1}=\left(s, f_{p}^{\infty}\right)$ which is a closed surjection. For $t<1, \Psi_{t}$ is onto since each component is onto by the Intermediate Value Theorem. Also, it is 
continuous, and hence $\Psi_{t}^{-1}$ is continuous since $\Psi_{t}$ is a closed map. It remains to show that for $t<1, \Psi_{t}$ is one-to-one. Let $(s, x),(s, y) \in Q^{2}$ be such that $\Psi_{t}(x)=\Psi_{t}(y)$. Then

$$
\left(s,(1-t) x+t f_{p}^{\infty}(x)\right)=\left(s,(1-t) y+t f_{p}^{\infty}(y)\right)
$$

and so

$$
(1-t) x+t f_{p}^{\infty}(x)=(1-t) y+t f_{p}^{\infty}(y) .
$$

It implies that $(1-t)(x-y)=t\left(f_{p}^{\infty}(y)-f_{p}^{\infty}(x)\right)$. If $x \neq y$, then there is $i$ such that $x_{i} \neq y_{i}$. Without loss of generality, assume that $x_{i}<y_{i}$. We know that $(1-t)\left(x_{i}-y_{i}\right)=t\left(f_{p}\left(y_{i}\right)-f_{p}\left(x_{i}\right)\right)$ Then the left hand side of equation is negative but the right hand side of equation is non-negative since the Cantor function $f_{p}$ is non-decreasing function. This leads to a contradiction. Thus $\Psi_{t}$ is one-to-one. Also, we can see that

$$
G_{f_{p}^{\infty}}^{s}=\left\{\Psi_{1}^{-1}(c) \mid c \in Q^{2}\right\} .
$$

The next lemma follows from the fact that $G_{f_{p}^{\infty}}^{s}$ is realized by a pseudoisotopy.

Lemma 15. Let $G_{f_{p}^{\infty}}^{s}$ be the decomposition of $Q_{s}^{2}=\{s\} \times Q_{2}$ induced by the map $f_{p}^{\infty}$. Then $\pi_{G_{p}^{\infty}}$ from $\{s\} \times Q_{2}$ to $\left(\{s\} \times Q_{2}^{s}\right) / G_{f_{p}^{\infty}}^{s}$ is approximable by homeomorphisms.

Lemma 16. The decomposition $G_{f_{p}^{\infty}}^{s}$ is cellular.

Proof. This follows from Lemma 12.

Next we will define a decomposition $G$ on $Q$. Given $s \in(0,1)$. Let $G$ be the partition consisting of $G_{f_{p}^{\infty}}^{s}=\left\{\{s\} \times\left(f_{p}^{\infty}\right)^{-1}(c) \mid c \in Q_{2}\right\}$ and all singletons in $Q-\{s\} \times Q_{2}$. It is clear that $G$ is a usc decomposition of $Q$ by a similar idea as shown in Lemma 13. Next we also show that $G$ is realized by a pseudoisotopy. First, for convenience, denote $\Psi_{t}^{o}(x)=(1-t) x+t f^{\infty}(x)$ the second component of $\phi_{t}(s, x)$ defined in the previous section. Also, $\Phi_{t}^{o}(x)$ is one-to-one since $\Psi_{t}(s, x)$ is one-to-one. Then define $K_{t}^{s}:[0,1] \times Q_{2} \rightarrow[0,1] \times Q_{2}$ by

$$
K_{t}^{s}(r, x)= \begin{cases}\left(r,(s-r) x+(1+r-s) \Psi_{t}^{o}(x)\right) & \text { if } 0 \leq r \leq s \\ \left(r,(r-s) x+(1-r+s) \Psi_{t}^{o}(x)\right) & \text { if } s \leq r \leq 1\end{cases}
$$

Claim that for $t<1, K_{t}^{s}$ is homeomorphism. Clearly, $K_{t}^{s}$ is onto, continuous and $\left(K_{t}^{s}\right)^{-1}$ is continuous. It remains to show that $K_{t}^{s}$ is one-to-one. Suppose 
that $K_{t}^{s}(a, x)=K_{t}^{s}(b, y)$ for some $(a, x),(b, y) \in[0,1] \times Q_{2}$. Then by the definition of $K_{t}^{s}$ we have $a=b$. If $a, b \in[0, s]$, then

$$
(s-a) x+(1+a-s) \Psi_{t}^{o}(x)=(s-a) y+(1+a-s) \Psi_{t}^{o}(y) .
$$

Consider

$$
\begin{aligned}
\Psi_{t(1+a-s)}^{o}(x) & =(1-t(1+a-s)) x+t(1+a-s) f^{\infty}(x) \\
& =(s-a) x+(1+a-s) \Psi_{t}^{o}(x) \\
& =(s-a) y+(1+a-s) \Psi_{t}^{o}(y) \\
& =(1-t(1+a-s)) y+t(1+a-s) f^{\infty}(y) \\
& =\Psi_{t(1+a-s)}^{o}(y) .
\end{aligned}
$$

Since $t<1$ and $1+a-s \leq 1$, it forces $t(1+a-s) \neq 1$. This yields $\Psi_{t(1+a-s)}^{o}$ is one-to-one and hence $x=y$. Similarly, for $a, b \in[s, 1], K_{t}^{s}$ is one-to-one. Therefore, $K_{t}^{s}$ is one-to-one for all $t<1$. Moreover, we can see that for each $(r, x) \in([0, s) \cup(s, 1]) \times Q_{2}$,

$$
K_{1}^{s}(r, x)= \begin{cases}\left(r,(s-r) x+(1+r-s) f_{p}^{\infty}(x)\right) & \text { if } 0 \leq r<s \\ \left(r,(r-s) x+(1-r+s) f_{p}^{\infty}(x)\right) & \text { if } s<r \leq 1\end{cases}
$$

is one-to-one since $r \neq s$. Thus $\left(K_{1}^{s}\right)^{-1}(r, x)$ is singleton and if $(r, x) \in\{s\} \times Q_{2}$, $K_{1}(s, x)=\{s\} \times\left(f_{p}^{\infty}\right)^{-1}(x) \in G_{f_{p}^{\infty}}^{s}$. Thus,

$$
G=\left\{K_{1}^{-1}(c) \mid c \in[0,1] \times Q_{2}\right\}=S \cup G_{f_{p}^{\infty}}^{s}
$$

where $S$ is the set of all singleton in $([0, s) \cup(s, 1]) \times Q_{2}$. Therefore, we see that $G=G_{f_{p}^{\infty}}^{s} \cup S$ is realized by pseudo-isotopies $K_{t}^{s}$. Thus, we have the following lemma.

Lemma 17. The decomposition $G$ is realized by pseudo-isotopy.

The result of Lemma 17 gives the following main results.

\section{Main Results}

Theorem 18. Let $G$ be the decomposition defined as above. Then $\pi_{G}$ from $Q$ to $Q / G$ is approximable by homeomorphisms, and hence $Q / G \cong Q$.

Proof. This follows from Lemma 17. 
Lemma 19. The decomposition $G$ is cellular.

Proof. This follows from Lemma 12.

\section{Acknowledgment}

This research is supported by the Centre of Excellence in Mathematics, the Commission on Higher Education, Thailand.

\section{References}

[1] Zvonko Čerin, On cellular decompositions of Hilbert cube manifolds, 91 (1980), 47-69.

[2] Donald R. Chalice, A characterization of the cantor function, The American Mathematical Monthly, 98 (1991), 255-258.

[3] T.A. Chapman, Lectures on Hilbert Cube Manifolds, CBMS Regional Conference Series in Mathematics (1976).

[4] Robert J. Daverman, Decompositions of Manifolds, AMS Chelsea Publishing (2007).

[5] Jozef Doboš, The standard Cantor function is subadditive, Proceedings of the American Mathematical Society, 124 (1996), 3425-3426.

[6] O. Dovgoshey, O. Martio, V. Ryazanov, M. Vuorinen, The Cantor function, Expo. Math., 24 (2006), 1-37.

[7] Dennis J. Garity, Thin codimension-one decomposition of $\mathbb{R}^{n}$, Topology and its Applications, 42 (1991), 263-276.

[8] Joerg Mayer, Algebraic Topology, Prentice-Hall, Inc. Engglewood Cliffs, NJ (1972).

[9] J.R. Munkres, Topology, Prentice Hall (2000).

[10] Aniruth Phon-On, A Thin Codimension-One Decomposition of the Hilbert Cube Q, Ph.D. Dissertation (2010).

[11] Stephen Willard, General Topology, Dover Publications, Inc. Mineola, NJ (1970). 